\title{
Central Nervous System Control of Glucose Homeostasis
}

\author{
Poondy Gopalratnam Raman \\ Department of Medicine, MGM Medical College, Devi Ahilya University, Indore, India \\ Email: drpgraman@yahoo.com
}

How to cite this paper: Raman, P.G. (2017) Central Nervous System Control of Glucose Homeostasis. Open Journal of Endocrine and Metabolic Diseases, 7, 227-234. https://doi.org/10.4236/ojemd.2017.712020

Received: August 27, 2017

Accepted: December 26, 2017

Published: December 29, 2017

Copyright (C) 2017 by author and Scientific Research Publishing Inc. This work is licensed under the Creative Commons Attribution International License (CC BY 4.0).

http://creativecommons.org/licenses/by/4.0/

\begin{abstract}
Hypothalamus and brain stem play important roles in Glucose Homeostasis. There are two types of cells in the hypothalamus: Glucose excited (GE) and Glucose inhibited (GI). GE increases glucose concentration and GI decreases glucose concentration. They are located in ventromedial ( $\mathrm{VMH})$, arcuate, lateral, dorsomedial and paraventricular areas of hypothalamus. Nucleus of solitary tract, area postrema, dorsomedial nucleus of vagus and basolateral medulla are also related to glucose homeostasis. VMH contains sympathetic nucleus and upregulates plasma glucose and decreases hepatic glycogen, while lateral hypothalamus contains parasympathetic and down regulates plasma glucose. Through Glut-1, dependent transport glucose enters neurons and astrocytes. Glucose is metabolized and provides energy for GE and GI neurons. Their activity is guided by blood sugar level. Blood sugar level sends numerous signals through vagal pathway from periphery. Neuron astrocyte establishes via autonomic system connections with liver, pancreas, adrenal gland and maintains glucose homeostasis. Post prandial glucose levels are regulated by CNS.
\end{abstract}

\section{Keywords}

Hypothalamus, Ventromedial Nucleus, Arcuate Nucleus, Dorsomedial Nucleus, Glut-1, GE and GI Neurons

\section{Historical Background}

1849: Claud Bernard showed puncturing floor of the fourth ventricle in brain, produced hyperglycemia in a few days time.

1953: Jean Mayer described two types of cells GE/GI neurons existence in brain.

1965: Shimazu on rabbits showed that electrical stimulation of VMH upregu- 
lates plasma glucose.

1994: Borg showed that lesions of VMH produced hypoglycemia due to decrease of hormonal counter regulation.

\section{Introduction}

Homeostasis can be defined as a property of an organism or system that helps it maintain its parameters within a normal range of values. It is key to life, and failures in homeostasis can lead to diseases like hypertension and diabetes.

Hypothalamus and brain stem play important roles in Glucose Homeostasis [1]. Central nervous system regulates fat metabolism, food intake, body weight and glucose homeostasis. Some of the molecular defects in type-2 diabetes may reside in hypothalamus. There are two types of cells in the hypothalamus: Glucose excited (GE) and Glucose inhibited (GI). GE increases glucose concentration and GI decreases glucose concentration as shown in Table 1 [2] [3]. They are located in ventromedial (VMH), arcuate, lateral, dorsomedial and paraventricular areas of hypothalamus. Nucleus of solitary tract, dorsomedial nucleus of vagus and basolateral medulla is also related to glucose homeostasis. VMH contains sympathetic nucleus and upregulates plasma glucose and decreases hepatic glycogen, while lateral hypothalamus contains parasympathetic and down regulates plasma glucose.

After nutrient injection, glucose is absorbed and distributed in equal thirds to liver, skeleton muscle and adipocytes. Acetyl choline is released from vagus and others like VIP, gastrin release peptide, pituitary adenylate cyclate activated peptide and nitrous oxide are responsible for linking Central Nervous System to hepatic glucose production axis.

Sympathetic nerves are activated by stress, hypoglycemia and produce neurotransmitter, norepinephrine and epinephrine. They act on liver, increase glucose production and decrease glucose uptake by skeletal muscles. It plays an important role in hind brain induced suppression of hepatic glucose production (Table 2).

Table 1. Effect of hormones and others on autonomic system, plasma glucose and liver glycogen [3].

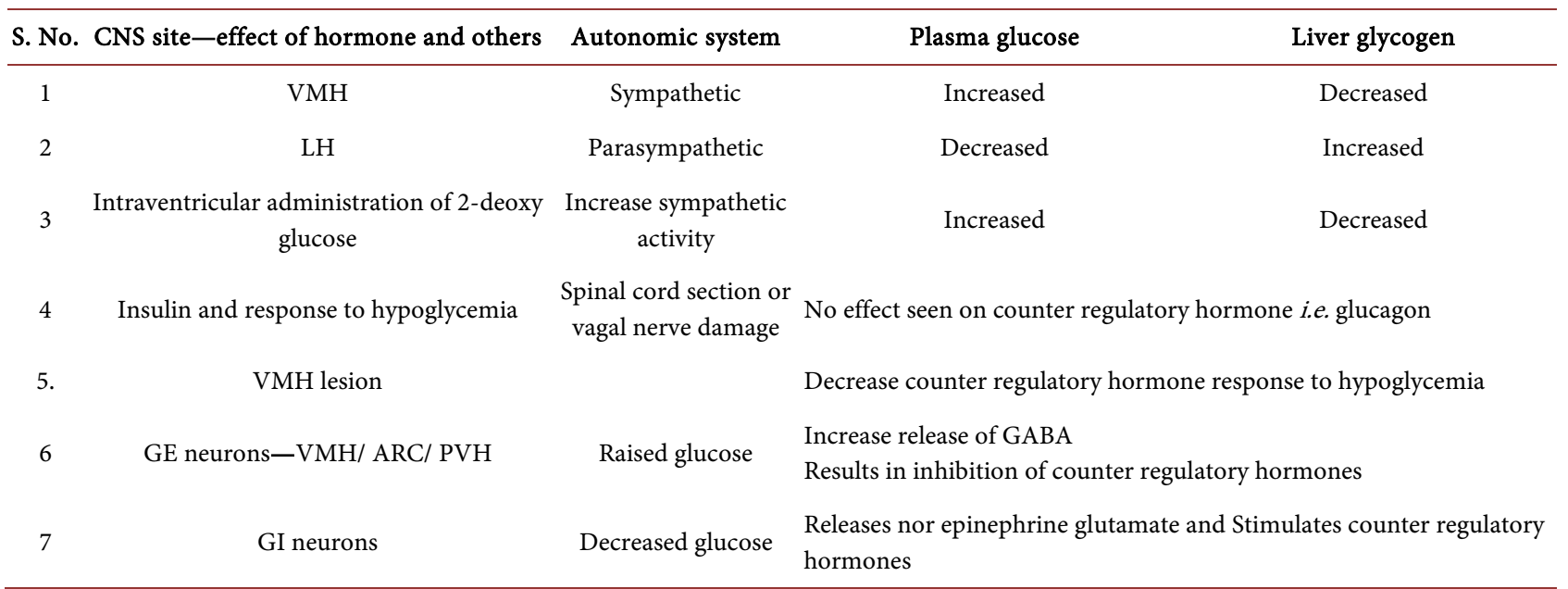


Table 2. Effect on Hepatic glucose production [18] [19].

\begin{tabular}{cl}
\hline \multicolumn{1}{c}{ Increased } & \multicolumn{1}{c}{ Decreased } \\
\hline Activation of central nor adrenergic, histaminergic, & Decreased by central cholinergic, muscarinic \\
serotonergic and cholinergic receptors and neurons & $\begin{array}{l}\text { receptors, histamine H1 receptors, } \mathrm{H} 2 \\
\text { receptors. }\end{array}$ \\
\hline
\end{tabular}

\section{Effect of High Fat Diet on Neuro-Gluco Regulation}

High fat diet leads to defective hormonal and nutrient sensing in peripheral organs leading to insulin resistance. Central nervous system regulation is impaired. Insulin, leptin and free fatty acid ability to reduce food intake is diminished.

Free fatty acid in CNS may come from local synthesis or from plasma, act on afferent signaling in glucose metabolism through Glucose sensing neurons in VMH or LH. Central injection of Oleic Acid decreases glucose output.

Circadian disruption leads to CAD, Alzheimer, CA, reproductive dysfunctions. Night shift workers exhibit shifted insulin glucose rhythms and have higher risk for development of type-2 diabetes compared to day shift workers [9] [10] [11]

It has long been known that sleep debt has a harmful impact on carbohydrate metabolism and endocrine function. It is therefore reasonable to expect that shift work may influence glucose tolerance. Alternating between day and night shifts resulting in continuous disruption of the body's circadian rhythms has been shown to be damaging to the health of workers. Shift work has a significantly detrimental effect on levels of hemoglobin Alc (HbAlc). The effect was similar to that seen with well-established risk factors, such as age and BMI.

The risk of diabetes, some studies reported an impairment of glucose tolerance in shift workers, with increased insulin resistance at night and a higher (almost double) prevalence of type 2 diabetes in relation to rotating shift work, which appears to increase with years spent doing shift work[12].

\section{Central Insulin Action [13]}

Insulin mediates direct effect, leading to inhibition of glycogenolysis and indirect effect through extra-hepatic action such as inhibition of lipolysis and reduction of glucagon levels. It activates hypothalamic insulin signaling and modulates feeding behavior, neuropeptide $\mathrm{Y}$ expression, hypoglycemia counter-regulation and autonomic outflow. At intracerebroventricular site, insulin down regulates glucose production. Leptin and insulin act on POMC, AgRP and VMH neurons and regulate glucose homeostasis in complicated manner.

Activation of insulin receptors in the hypothalamus in particular in the arcuate nucleus (ARC) plays an important role in regulation of glucose homeostasis. Activation of insulin signaling in ARC in the absence of elevated systemic insulin levels is sufficient to decrease blood glucose levels via substantial inhibition of endogenous glucose production. Conversely, the blockade of insulin action in ARC by insulin antibodies or by decreasing insulin receptors by antisense 
oligonucleotides or by inhibiting the insulin dependent activation of phosphatidylinositol-3 kinase leads to a reduced ability of circulating insulin to suppress endogenous glucose production.

\section{Arcuate Nucleus Centre Control of Blood Glucose [14] [15] [16]}

The activation of insulin signaling in hypothalamic arcuate nucleus (ARC) is sufficient to decrease blood glucose levels via the inhibition of hepatic glucose production (GP). This effect is mediated by the activation of efferent vagal fibers which innervate the liver. Leptin acts on hypothalamus at ARC, POMC, $\alpha$-MSH and agouti related peptides (AgRP) and decreases food intake. Leptin decreases glycogenolysis, increases gluconeogenesis [17].

Delivery of a KATP channel blocker to the ARC, such as glibenclamide, abolishes the central effects of insulin on endogenous glucose production, and prevents, in part, the suppression of endogenous glucose production by circulating insulin. Insulin acts on KATP channels in hypothalamic neurons to control hepatic glucose production by decreasing glucose-6-phosphatase and phosphoenolpyruvate kinase expression in the liver.

Glucagon-like peptide-1 (GLP-1) is produced in a discrete set of hindbrain neurons that project to a specific population of GLP-1 receptor-containing cells in the hypothalamus. During hyperglycemia following a meal, GLP-1 inhibits muscle glucose utilization and increases insulin secretion to favor hepatic glycogen storage. The intracellular pool of long-chain fatty acids (LCFA-CoAs) is under the control of several biochemical events including a free fatty acids (FFA) flux. Accumulation of LCFA-CoAs reduces hepatic glucose production.

\section{Central Leptin Actions [13]}

Leptin is a hormone produced by white adipose tissue that has potent effects on feeding behavior, thermogenesis and neuroendocrine responses. The severe obesity caused by leptin absence in rodents and humans makes it clear that leptin is a fundamental hormone regulating energy homeostasis. Abundant evidence, including the brain-specific knockout of the leptin receptor, indicates that the CNS is the site for leptin anti-obesity actions. The leptin receptor mRNA is highly expressed in the hypothalamus, including the ARC, the ventromedial nucleus and the dorsomedial nucleus. Leptin administration has impressive effects in improving insulin action and glucose homeostasis.

The effects of central leptin on glucose homeostasis involve both melanocortin dependent and melanocortin-independent pathways. Acute activation of hypothalamic melanocortin receptors, results in an increased rate of hepatic gluconeogenesis. The effect of central leptin on the suppression of glucose production and glycogenolysis is mediated by the stimulation of melanocortin-independent pathways involving insulin-like pathways such as the PI3K signaling cascade.

Restoration of leptin receptors in the ARC had modest effects on body weight, 
but remarkably improved glucose homeostasis. Melanocortin signaling acutely affects insulin levels and glucose uptake, and humans with MC4R mutations are extremely insulin resistant. Acute activation of hypothalamic melanocortin receptors, results in an increased rate of hepatic gluconeogenesis and the increased expression of the rate-limiting gluconeogenic enzymes phosphoenolpyruvate carboxykinase (PEPCK).

\section{Central GLP-1 Actions}

Besides the gut, the other major site of GLP-1 production is the brain. GLP-1 is produced in a discrete set of hindbrain neurons that project to a specific population of GLP-1 receptor-containing cells in the brain stem, hypothalamus and midbrain. The CNS and peripheral actions of GLP-1 are distinct and independent. GLP-1 signaling plays a role in the control of blood glucose levels.

GLP-1 activity in the brain induces peripheral neural signals which regulate systemic glucose metabolism. Interestingly, when the CNS GLP-1 receptors were blocked, there was a significant attenuation of meal-induced insulin secretion.

\section{Neuronal Biochemical Sensors}

The central administration of macronutrients such as glucose or oleic acid decreases blood glucose and insulin levels. Like central insulin action, the central effect of circulating macronutrients on glucose production is mediated by the activation of KATP channels in the ARC. Elevations of plasma levels of free fatty acids (FFA) or glucose (Table 3), activate hypothalamic centers which in turn suppress endogenous glucose production.

Neuronal levels of malonyl-CoA act as neural sensors of fuel availability and regulators of energy balance and glucose homeostasis.

Evidence suggests two gut peptides secreted post-prandially viz. CCK and GLP-1 act via vagal afferents to regulate glucose homeostasis forming a gut to brain to liver axis. It helps in suppression of hepatic glucose production.

Bariatric surgery is one form of combined therapy for obesity and T2DM. RYGM and VSG are now known as metabolic surgeries. Duodeno jejunal bypass has shown to lower glucose by increasing nutrient flow across jejunum. Surgery leads to increase in CCK, GLP-1 and circadian rhythm is corrected.

Table 3. Effect of high fat diet on glucose regulation.

\begin{tabular}{|c|c|c|}
\hline S.No. & Site (activation of hypothalamus) & Effect \\
\hline 1 & Inflammatory pathway & Insulin resistance and leptin resistance \\
\hline 2 & $\begin{array}{c}\text { Melanocortin } 4 \text { receptor (MC4R) } \\
\text { resistance }\end{array}$ & Neuronal circulatory downstream of ARC \\
\hline 3 & Leptin resistance & $\begin{array}{l}\text { Anorectic action blunted and lowers } \\
\text { gluconeogenesis }\end{array}$ \\
\hline 4 & HGP (hepatic glucose production) & Hypothalamic sensing of nutrients blunted \\
\hline
\end{tabular}


Table 4. Circadian rhythm (brain clock) [4] [5] [6] [7] [8].

\begin{tabular}{lclll}
\hline & Plasma & Morning & Afternoon & Evening \\
\hline 1. & Plasma glucose & Decreased & Increased & Increased \\
2. & Insulin level & Increased & Increased & Increased \\
3. & Skeletal muscle glucose uptake & Increased & Increased & Increased \\
4. & HGP (hepatic glucose production) & Increased & Increased & Increased \\
\hline
\end{tabular}

Peripheral stimulus - Taste receptor - Hepatoportal receptor - Gut receptor - Blood glucose level $\downarrow$

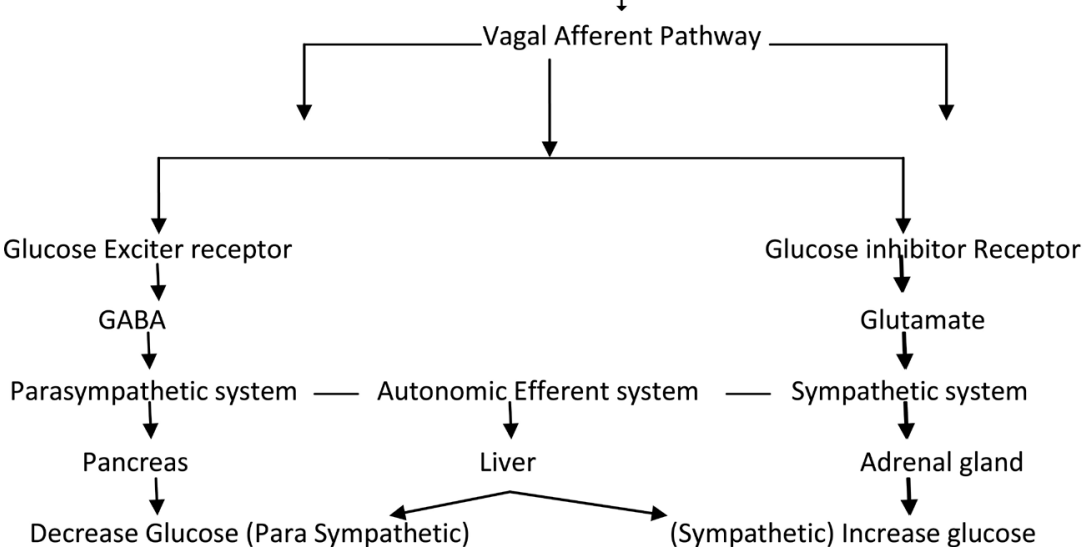

\section{Drugs/Hormones Working through Circadian Rhythm of Brain (Table 4)}

1) Melatonin increases TG clearance.

2) Quick release Bromocriptine to be taken in the morning on waking up, is used in T2DM treatment.

3) Increased hypothalamic noradrenergic neuronal activity leads to hyperglycemia.

\section{Conclusion}

The hypothalamus is emerging as a critical site for the integration of nutritional, endocrine, and neural cues signaling the body's metabolic and nutritional status. Onset of hypothalamic resistance to multiple signals, such as leptin, insulin, and fatty acids, could contribute to the susceptibility to weight gain and insulin resistance in predisposed individuals. Through Glut-1 dependent transport glucose enters neurons and astrocytes. Glucose is metabolized and provides energy for GE and GI neurons. Their activity is guided by blood sugar level. Blood sugar level sends numerous signals through vagal pathway from periphery. Neuron astrocyte establishes via autonomic system connections with liver, pancreas, adrenal gland and maintains glucose homeostasis. Post prandial glucose levels are also regulated by CNS. Current therapies in T2DM are peripherally directed. We must target therapies on CNS primary site of dysfunction for both obesity and T2DM. In addition, the hypothalamic circuits that regulate insulin action might become the targets of future preventive and therapeutic strategies. 


\section{References}

[1] Lam, C.K., Chari, M. and Lam, T.K. (2009) CNS Regulation of Glucose Homeostasis. Physiology, 24, 159-170. https://doi.org/10.1152/physiol.00003.2009

[2] Muntean, C., Mota, M. and Popa, S. and Mitrea, S. (2012) The Role of the Central Nervous System in Glucose Homeostasis. Romanian Journal of Diabetes Nutrition \& Metabolic Diseases, 19, 207-214.

[3] Grayson, B.E., Seeley, R.J. and Sandoval, D.A. (2013) Wired on Sugar: The Role of the CNS in the Regulation of Glucose Homeostasis. Nature Reviews Neuroscience, 14, 24-37. https://doi.org/10.1038/nrn3409

[4] Takahashi, J.S., Hong, H.-K., Ko, C.H. and McDearmon, E.L. (2008) The Genetics of Mammalian Circadian Order and Disorder: Implications for Physiology and Disease. Nature Reviews Genetics, 9, 764-775. https://doi.org/10.1038/nrg2430

[5] Yamazaki, S., Numano, R., Abe, M., et al. (2000) Resetting Central and Peripheral Circadian Oscillators in Transgenic rats. Science, 288, 682-685. https://doi.org/10.1126/science.288.5466.682

[6] Van Cauter, E., Polonsky, K.S. and Scheen, A.J. (1997) Roles of Circadian Rhythmicity and Sleep in Human Glucose Regulation. Endocrine Reviews, 18, 716-738.

[7] Coomans, C.P., van den Berg, S.A.A., Lucassen, E.A., et al. (2013) The Suprachiasmatic Nucleus Controls Circadian Energy Metabolism and Hepatic Insulin Sensitivity. Diabetes, 62, 1102-1108. https://doi.org/10.2337/db12-0507

[8] Damiola, F., Le Minh, N., Preitner, N., Kornmann, B., Fleury-Olela, F. and Schibler, U. (2000) Restricted Feeding Uncouples Circadian Oscillators in Peripheral Tissues from the Central Pacemaker in the Suprachiasmatic Nucleus. Genes \& Development, 14, 2950-2961. https://doi.org/10.1101/gad.183500

[9] Van Cauter, E. (2011) Sleep Disturbances and Insulin Resistance. Diabetic Medicine, 28, 1455-1462. https://doi.org/10.1111/j.1464-5491.2011.03459.x

[10] Morris, C.J., Aeschbach, D. and Scheer, F.A.J.L. (2012) Circadian System, Sleep and Endocrinology. Molecular and Cellular Endocrinology, 349, 91-104. https://doi.org/10.1016/j.mce.2011.09.003

[11] Lauer, C.J. and Krieg, J.-C. (2004) Sleep in Eating Disorders. Sleep Medicine Reviews, 8, 109-118. https://doi.org/10.1016/S1087-0792(02)00122-3

[12] Costa, G. (2010) Shift Work and Health: Current Problems and Preventive Actions. Safety Health Work, 1, 112-123. https://doi.org/10.5491/SHAW.2010.1.2.112

[13] Puglianiello, A. and Cianfarani, S. (2006) Central Control of Glucose Homeostasis. The Review of Diabetic Studies, 3, 54-60. https://doi.org/10.1900/RDS.2006.3.54

[14] Kalsbeek, A., Yi, C.X., La Fleur, S.E. and Fliers, E. (2010) The Hypothalamic Clock and Its Control of Glucose Homeostasis. Trends in Endocrinology \& Metabolism, 21, 402-410. https://doi.org/10.1016/j.tem.2010.02.005

[15] Delezie, J. and Challet, E. (2011) Interactions between Metabolism and Circadian Clocks: Reciprocal Disturbances. Annals of the New York Academy of Sciences, 1243, 30-46. https://doi.org/10.1111/j.1749-6632.2011.06246.x

[16] Sahar, S. and Sassone-Corsi, P. (2012) Regulation of Metabolism: The Circadian Clock Dictates the Time. Trends in Endocrinology \& Metabolism, 23, 1-8. https://doi.org/10.1016/j.tem.2011.10.005

[17] Morton, G.J. and Schwartz, M.W. (2011) Leptin and the CNS Control of Glucose Metabolism. Physiological Reviews, 91, 389-411. https://doi.org/10.1152/physrev.00007.2010 
[18] Mendoza, J. (2007) Circadian Clocks: Setting Time by Food. Journal of Neuroendocrinology, 19, 127-137. https://doi.org/10.1111/j.1365-2826.2006.01510.x

[19] Carniero, B.T.S. and Araujo, J.F. (2012) Food Entrainment: Major and Recent Findings. Frontiers in Behavioral Neuroscience, 6, 1-6.

https://doi.org/10.3389/fnbeh.2012.00083

\section{Abbreviations}

PVH: Paraventricular nucleus of hypothalamus

GE: $\quad$ Glucose excited cells

GI: $\quad$ Glucose inhibited cells

VMH: Ventromedial hypothalamus

ARC: Arcuate Nucleus Centre

POMC: Pro-opiomelanocortin

$\alpha$-MSH: Alpha-Melanocyte stimulating hormone

AGRP: Agouti-related peptide

LH: Luteinizing hormone (Leptin hormone)

VIP: Vasoactive intestinal peptide

FFA: $\quad$ Free fatty acid

CAD: Coronary artery disease

CA: Cancer

CCK: Cholecystokinin

GLP-1: Glucagon-like peptide-1

TG: Triglyceride

T2DM: Type-2 Diabetes

MC4R: Melanocortin 4 receptor 